\title{
Local Existence of Solution to a Class of Stochastic Differential Equations with Finite Delay in Hilbert Spaces
}

\author{
Le Anh Minh, Hoang Nam, Nguyen Xuan Thuan \\ Department of Natural Sciences, Hong Duc University, Thanh Hoa, Vietnam \\ Email: leanhminh@hdu.edu.vn, hoangnam@hdu.edu.vn, thuannx7@gmail.com
}

Received October 6, 2012; revised November 6, 2012; accepted November 14, 2012

\begin{abstract}
In this paper, we present a local Lipchitz condition for the local existence of solution to a class of stochastic differential equations with finite delay in a real separable Hilbert space which has the following form:

$d X(t)=A X(t)+f\left(t, X_{t}\right) d t+g\left(t, X_{t}\right) d W(t), \quad t \geq 0$
\end{abstract}

Keywords: Stochastic Differential Equation; Local Lipchitz Condition; Strongly Semigroup

\section{Introduction}

The purpose of this paper focuses on the local existence of mild solution to a class of the following stochastic differential equations with finite delay in a real separable Hilbert space $H$

$$
\begin{aligned}
& d X(t)=A X(t)+f\left(t, X_{t}\right) d t+g\left(t, X_{t}\right) d W(t), \\
& t \geq 0
\end{aligned}
$$

where $A: \mathcal{D}(A) \subset H \rightarrow H$ is a linear (possibly unbound) operator, and with a constant $\tau>0$ we define

$$
\begin{aligned}
X_{t} \in C_{r}:=C([-\tau, 0], H) \text { by } \\
\\
X_{t}(\theta)=X(t+\theta), \theta \in[-\tau, 0]
\end{aligned}
$$

In which, $C_{r}$ is the space of all continuous functions from $[-\tau, 0]$ into $H$ equipped with the norm

$$
\|z\|_{C_{r}}=\left(\sup _{-\tau \leq \theta \leq 0}\|z(\theta)\|_{H}^{2}\right)^{1 / 2} \text {. }
$$

$\left(f: \mathbb{R}_{+} \times C_{r} \rightarrow H\right.$ and $g: \mathbb{R}_{+} \times C_{r} \rightarrow L_{2}^{0}$ are continuous functions; $W(t)$ is a $\mathcal{Q}$-Weiner process defined in Section 2).

In [1], if $A$ is the generator of a uniformly exponentially stable semi-group in $H ; f, g$ satisfies Lipchitz and linear growth conditions then the mild solution of Equation (1) is exponentially stable in mean square.

In this paper, we prove the local existence of solution for Equation (1) with the weaker condition on $A, f$; and $g$.

\section{Preliminaries}

In this section, we will recall some notions from Bezandry and Diagana [1].
Let $H, K$ be real separable Hilbert spaces, $\left(\Omega, F, P, \mathcal{F}_{t}\right)$ be a filtered probability space; and $b_{n}(t), n=1,2, \cdots$ is a sequence of real-valued standard Brownian motions mutually independent on this space. Furthermore,

$$
W(t)=\sum_{n=1}^{\infty} \sqrt{\lambda_{n}} b_{n} e_{n}, \quad t \geq 0 .
$$

where $\lambda_{n} \geq 0,(n \geq 1)$ are nonnegative real numbers; and $\left(e_{n}\right)_{n \geq 1}$ is the complete orthonormal basis in $K$.

In addition, we suppose that $\mathcal{Q} \in B(K, K)$ is an operator defined by $\mathcal{Q} e_{n}=\lambda_{n} e_{n}$ such that

$$
\operatorname{Tr} \mathcal{Q}=\sum_{n=1}^{\infty} \lambda_{n}<\infty .
$$

Then, $E W(t)=0$ and for all $t \geq s \geq 0$ the distribution of $W(t)-W(s)$ is $\mathcal{N}(0,(t-s) \mathcal{Q})$. The $K$-valued stochastic process $W(t)$ is called a $\mathcal{Q}$-Weiner process.

The subset $K_{0}=\mathcal{Q}^{1 / 2} K$ is a Hilbert space equipped with the norm

$$
\|u\|_{K_{0}}=\left\|\mathcal{Q}^{1 / 2} u\right\|_{K}, u \in K_{0}
$$

and we define the space of all Hilbert-Schmidt operators from $K_{0}$ into $H$ by

$$
\begin{aligned}
& L_{2}^{0}=L_{2}^{0}\left(K_{0}, H\right) \\
& =\left\{\psi \in B\left(K_{0}, H\right): \operatorname{Tr}\left[\left(\psi Q^{1 / 2}\right)\left(\psi Q^{1 / 2}\right)^{*}\right]<\infty\right\}
\end{aligned}
$$

Clearly, $L_{2}^{0}$ is a separable Hilbert space with norm

$$
\|\psi\|_{L_{2}^{0}}^{2}=\operatorname{Tr}\left[\left(\psi Q^{1 / 2}\right)\left(\psi Q^{1 / 2}\right)^{*}\right], \psi \in L_{2}^{0} .
$$

Let $\mathcal{U}^{2}\left([0, T], L_{2}^{0}\right)$ be all $L_{2}^{0}$ - valued predictable 
processes $\Phi$ such that

$$
E \int_{0}^{T} \operatorname{Tr}\left[\left(\psi Q^{1 / 2}\right)\left(\psi Q^{1 / 2}\right)^{*}\right] \mathrm{d} s<\infty .
$$

Then, for all $\Phi \in \mathcal{U}^{2}\left([0, T], L_{2}^{0}\right)$ the stochastic integral $\int_{0}^{t} \Phi(s) \mathrm{d} W(s) \in H$ is well-defined by

$$
\begin{aligned}
& \int_{0}^{t} \Phi(s) \mathrm{d} W(s)=\lim _{n \rightarrow \infty} \sum_{i=0}^{n} \int_{0}^{t} \Phi(s) \sqrt{\lambda_{i}} e_{i} \mathrm{~d} b_{i}(s), \\
& t \in[0, T]
\end{aligned}
$$

where $W$ is the $\mathcal{Q}$-Weiner process defined above. We have

$$
\begin{aligned}
& E\left\|\int_{0}^{t} \Phi(t) \mathrm{d} W(s)\right\|_{H}^{2} \leq E \int_{0}^{t}\|\Phi(s)\|_{L_{2}^{0}}^{2} \mathrm{~d} s, \\
& 0 \leq t \leq T .
\end{aligned}
$$

In the following, we assume the stochastic integrals are well defined. For stochastic differential equation and stochastic calculus, we refer to [1-8].

\subsection{Definition [1]}

For $T \geq 0$, a stochastic process $X(t)$ is said to be a strong solution of Equation (1) on $[-r, T]$ if

1) $X(t)$ is adapted to $\mathcal{F}_{t}$ for all $t \geq 0$;

2) $X(t)$ is continuous in $t$ almost sure;

3) $X(t) \in D(A)$ for any $t \geq 0, \int_{0}^{t}\|A X(s)\| \mathrm{d} s<\infty$ almost surely for any $t>0$, and

$$
\begin{aligned}
& X(t)=X(0)+\int_{0}^{t} A X(s) \mathrm{d} s \\
& +\int_{0}^{t} f\left(s, X_{s}\right) \mathrm{d} s+\int_{0}^{t} g\left(s, X_{s}\right) \mathrm{d} W(s)
\end{aligned}
$$

for all $t \geq 0$ with probability one.

4) $X(t)=\varphi(t),-r \leq t \leq 0$ almost surely.

\subsection{Definition [1]}

For $T \geq 0$, a stochastic process $X(t)$ is said to be a mild solution of Equation (1) on $[-r, T]$ if

1) $X(t)$ is adapted to $\mathcal{F}_{t}$ for all $t \geq 0$;

2) $X(t)$ is continuous in $t$ almost sure;

3) $X$ is measureable with $\int_{0}^{T}\|X(t)\|^{2} \mathrm{~d} t<\infty$ almost surely for any $T>0$ and

$$
\begin{aligned}
X(t, \varphi)= & T(t) \varphi(0)+\int_{0}^{t} T(t-s) f\left(s, X_{s}\right) \mathrm{d} s \\
& +\int_{0}^{t} T(t-s) g\left(s, X_{s}\right) \mathrm{d} W(s)
\end{aligned}
$$

for all $t \geq 0$ with probability one;
4) $X(t)=\varphi(t),-r \leq t \leq 0$ almost surely.

\section{Main Results}

We assume that

$\left(^{*}\right)$ The operator $A$ generates a strongly semi-group $(T(t))_{t \geq 0}$ in $H$.

(**) $f(t, x)$ and $g(t, x)$ satisfy local Lipchitz conditions respects to second argument that means for $\alpha>0$ be a given real number, there exits $C_{1}(\alpha), C_{2}(\alpha)>0$ such that with $t \geq 0, \quad x, y \in C_{r}$, and $\|x\|,\|y\| \leq \alpha$, we have

$$
\begin{aligned}
& \|f(t, x)-f(t, y)\| \leq C_{1}(\alpha)\|x-y\|_{C_{r}}, \\
& \|g(t, x)-g(t, y)\|_{L_{2}^{0}} \leq C_{2}(\alpha)\|x-y\|_{C_{r}} .
\end{aligned}
$$

If condition (*) holds, we will prove that if $X(t)$ is a strong solution of Equation (1) then it also is a mild one. It is expressed by Theorem 3.1.

\subsection{Theorem}

If $(*)$ holds then (3) can be written in the form (4).

Proof: Applying Fubini theorem, we have

$$
\begin{aligned}
& \int_{0}^{t} T(t-s) \int_{0}^{s} g\left(u, X_{u}\right) \mathrm{d} W(u) \mathrm{d} s \\
& =\int_{0}^{t} \int_{u}^{t} T(t-s) g\left(u, X_{u}\right) \mathrm{d} s \mathrm{~d} W(u) \text { a.e. }
\end{aligned}
$$

On the other hand

$$
\begin{aligned}
& A \int_{u}^{t} T(t-s) g\left(s, X_{s}\right) \mathrm{d} s \mathrm{~d} W(u) \\
& =\int_{0}^{t} A \int_{0}^{t-u} T(s) g\left(s, X_{s}\right) \mathrm{d} s \mathrm{~d} W(u) \\
& =\int_{0}^{t}(T(t-s)-I) g\left(s, X_{s}\right) \mathrm{d} W(s) \text { a.e }
\end{aligned}
$$

From (5) and (6), one has

$$
\begin{aligned}
& A \int_{0}^{t} T(t-s) \int_{0}^{s} g\left(s, X_{s}\right) \mathrm{d} W(u) \mathrm{d} s \\
& =\int_{0}^{t}(T(t-s)-I) g\left(s, X_{s}\right) \mathrm{d}(s) \quad \text { a.e }
\end{aligned}
$$

or

$$
\begin{aligned}
\int_{0}^{t} g\left(s, X_{s}\right) \mathrm{d}(s) & =\int_{0}^{t} T(t-s) g\left(s, X_{s}\right) \mathrm{d} W(s) \\
& -A \int_{0}^{t} T(t-s) \int_{0}^{s} g\left(s, X_{s}\right) \mathrm{d} W(u) \mathrm{d} s .
\end{aligned}
$$


By the definition of strong solution, we have

$$
\begin{aligned}
& X(t)-\varphi(0)-A \int_{0}^{t} X(s) \mathrm{d} s-\int_{0}^{t} f\left(s, X_{s}\right) \mathrm{d} s \\
& =\int_{0}^{t} g\left(s, X_{s}\right) \mathrm{d} W(s) \\
& =\int_{0}^{t} T(t-s) g\left(s, X_{s}\right) \mathrm{d} W(s) \\
& -A \int_{0}^{t} T(t-s) \int_{0}^{s} g\left(s, X_{s}\right) \mathrm{d} W(u) \mathrm{d} s
\end{aligned}
$$

Since

$$
\begin{aligned}
& \int_{0}^{t} T(t-s) \int_{0}^{s} g\left(s, X_{s}\right) \mathrm{d} W(u) \mathrm{d} s \\
& =\int_{0}^{t} T(t-s)\left[X(s)-\varphi(0)-A \int_{0}^{s} X(u) \mathrm{d} u-\int_{0}^{s} f\left(u, X_{u}\right) \mathrm{d} u\right] \mathrm{d} s \\
& =\int_{0}^{t} T(t-s) X(s) \mathrm{d} s-\int_{0}^{t} T(t-s) \varphi(0) \mathrm{d} s \\
& -A \int_{0}^{t} \int_{u}^{t} T(t-s) X(u) \mathrm{d} s \mathrm{~d} u \\
& \quad-\int_{0}^{t} T(t-s) \int_{u}^{t} f\left(u, X_{u}\right) \mathrm{d} s \mathrm{~d} u \\
& =-\int_{0}^{t} T(t-s) \varphi(0) \mathrm{d} s+\int_{0}^{t} X(s) \mathrm{d} s
\end{aligned}
$$

We have

$$
\begin{aligned}
& A \int_{0}^{t} T(t-s) \int_{0}^{s} g\left(s, X_{s}\right) \mathrm{d} W(u) \mathrm{d} s \\
& =-(T(t) \varphi(0)-\varphi(0))+A \int_{0}^{t} X(s) \mathrm{d} s \\
& -\left(\int_{0}^{t} T(t-s) f\left(s, X_{s}\right) \mathrm{d} s-\int_{0}^{t} f\left(s, X_{s}\right) \mathrm{d} s\right) .
\end{aligned}
$$

Substituting equation above for (8), we received

$$
\begin{aligned}
& X(t)-\varphi(0)-A \int_{0}^{t} X(s) \mathrm{d} s-\int_{0}^{t} f\left(s, X_{s}\right) \mathrm{d} s \\
& =\int_{0}^{t} T(t-s) g\left(s, X_{s}\right) \mathrm{d} W(s)+T(t) \varphi(0)-\varphi(0) \\
& -A \int_{0}^{t} X(s) \mathrm{d} s+\int_{0}^{t} T(t-s) f\left(s, X_{s}\right) \mathrm{d} s-\int_{0}^{t} f\left(s, X_{s}\right) \mathrm{d} s .
\end{aligned}
$$

Hence,

$$
\begin{aligned}
X(t)= & T(t) \varphi(0)+\int_{0}^{t} T(t-s) f\left(s, X_{s}\right) \mathrm{d} s \\
& +\int_{0}^{t} T(t-s) g\left(s, X_{s}\right) \mathrm{d} W(s)
\end{aligned}
$$

Now, we turn our attention to the local existence of mild solution of Equation (1).

\subsection{Theorem}

If the condition $(*)$ and $\left({ }^{* *}\right)$ are satisfied, then (1) has only mild solution.

Proof: Let $T>0$ be a fixed number in $\mathbb{R}$, for each $\alpha>0$, there exists $\varphi \in C_{r} \quad(\|\varphi\| \leq \alpha)$, such that

$$
\begin{aligned}
\|f(t, \varphi)\| & \leq C_{1}(\alpha)\|\varphi\|+\|f(t, 0)\| \\
& \leq \alpha C_{1}(\alpha)+\sup _{s \in[0, T]}\|f(s, 0)\| \leq C, \\
\|g(t, \varphi)\| & \leq C_{2}(\alpha)\|\varphi\|+\|g(t, 0)\| \\
& \leq \alpha C_{2}(\alpha)+\sup _{s \in[0, T]}\|g(s, 0)\| \leq C,
\end{aligned}
$$

where

$$
\begin{array}{r}
C=\max \left\{\alpha C_{1}(\alpha)+\sup _{s \in[0, T]}\|f(s, 0)\|,\right. \\
\left.\alpha C_{2}(\alpha)+\sup _{s \in[0, T]}\|g(s, 0)\|\right\} .
\end{array}
$$

For any $\varphi \in C_{r}$, we chose $\alpha=\|\varphi\|+1$. Let $C_{a d}$ be a subspace of $C([-r, T], H)$ containing all functions $X$ which adapt with $\left\{F_{t}\right\}_{t \geq 0}$ such that $X_{0} \in C_{r}$ and $X:[0, T] \rightarrow H$ is continuous. Then $C_{a d}$ is a Banach space with norm

$$
\|X\|_{a d}=\left\|X_{0}\right\|_{C_{r}}+\max _{0 \leq t \leq T}\left(E\|X(t)\|^{2}\right)^{1 / 2} .
$$

Let us consider a set $\mathrm{Z}$ which is defined by

$$
\begin{aligned}
Z= & \left\{X \in C_{a d}: X(s)=\varphi(s) \text { for } s \in[-r, 0]\right. \\
& \text { and } \left.\sup _{0 \leq s \leq T}\|X(s)-\varphi(0)\| \leq 1\right\}
\end{aligned}
$$

It is easy to verify that $Z$ is a closed subspace of $C_{a d}$.

Let $U: Z \rightarrow Z$ be the operator defined by

$$
U(X)(t)= \begin{cases}T(t) \varphi(0)+\int_{0}^{t} T(t-s) f\left(s, X_{s}\right) \mathrm{d} s \\ +\int_{0}^{t} T(t-s) g\left(s, X_{s}\right) \mathrm{d} W(s) & \text { for } t \in[0, T] \\ \varphi(t) & \text { for } t \in[-r, 0]\end{cases}
$$

We now prove that $U(Z) \subseteq Z$. Indeed, 


$$
\begin{aligned}
& \|U(X)(t)-\varphi(0)\|^{2}=E\|U(X)(t)-\varphi(0)\|^{2}=E\left(\left\|T(t) \varphi(0)-\varphi(0)+\int_{0}^{t} T(t-s) f\left(s, X_{s}\right) \mathrm{d} s+\int_{0}^{t} T(t-s) g\left(s, X_{s}\right) \mathrm{d} W(s)\right\| \|^{2}\right. \\
& \leq 3 E\|T(t) \varphi(0)-\varphi(0)\|^{2}+3 E\left\|\int_{0}^{t} T(t-s) f\left(s, X_{s}\right) \mathrm{d} s\right\|^{2}+3 E\left\|\int_{0}^{t} T(t-s) g\left(s, X_{s}\right) \mathrm{d} W(s)\right\|^{2} \\
& \leq 3 E\|T(t) \varphi(0)-\varphi(0)\|^{2}+3 M T \int_{0}^{t} E\left\|f\left(s, X_{s}\right)\right\|^{2} \mathrm{~d} s+3 M \int_{0}^{t} E\left\|g\left(s, X_{s}\right)\right\|_{L_{2}^{0}}^{2} \mathrm{~d} s .
\end{aligned}
$$

Since $\|X(s)-\varphi(0)\| \leq 1, \forall s \in[0, T],\|X(s)\| \leq \alpha$ with $\alpha=\|\varphi\|+1$, we have $\left\|X_{s}\right\| \leq \alpha$ for any $s \in[0, T]$.

Furthermore,

$$
\left\|f\left(s, X_{s}\right)\right\| \leq C \text { and }\left\|g\left(s, X_{s}\right)\right\| \leq C .
$$

Hence

$$
\begin{aligned}
& \|U(X)(t)-\varphi(0)\|^{2} \\
& \leq 3 E\|T(t) \varphi(0)-\varphi(0)\|^{2}+3 M C^{2}\left(T^{2}+T\right)
\end{aligned}
$$

with $M=\sup _{0 \leq t \leq T}\|T(t)\|^{2}$.

If we choose $T$ small enough, such that

$$
\sup _{0 \leq s \leq T}\left\{3 E\|T(s) \varphi(0)-\varphi(0)\|^{2}+3 M C^{2}\left(T^{2}+T\right)\right\} \leq 1 .
$$

Then, for any $t \in[0, T]$ we have $\|U(X)(t)-\varphi(0)\| \leq 1$. In other words, we have $U(Z) \subseteq Z$.

For any $X, Y \in Z$,

$$
\begin{aligned}
& E\|U(X)(t)-U(Y)(t)\|^{2} \\
& =E \| \int_{0}^{t} T(t-s)\left[f\left(s, X_{s}\right)-f\left(s, Y_{s}\right)\right] \mathrm{d} s \\
& +\int_{0}^{t} T(t-s)\left[g\left(s, X_{s}\right)-g\left(s, Y_{s}\right)\right] \mathrm{d} W(s) \|^{2} \\
& \leq 2 E\left(\int_{0}^{t}\left\|T(t-s)\left[f\left(s, X_{s}\right)-f\left(s, Y_{s}\right)\right]\right\| \mathrm{d} s\right)^{2} \\
& +2 E\left(\int_{0}^{t}\left\|T(t-s)\left[g\left(s, X_{s}\right)-g\left(s, Y_{s}\right)\right] \mathrm{d} W(s)\right\|\right)^{2} \\
& \leq 2 M E\left(\int_{0}^{t}\left\|f\left(s, X_{s}\right)-f\left(s, Y_{s}\right)\right\| \mathrm{d} s\right)^{2} \\
& +2 M E\left(\int_{0}^{t}\left\|g\left(s, X_{s}\right)-g\left(s, Y_{s}\right)\right\| \mathrm{d} W(s)\right)^{2} \\
& \leq 2 M C^{2} T \int_{0}^{t} E\|X(s)-Y(s)\|^{2} \mathrm{~d} s \\
& +2 M C^{2} \int_{0}^{t} E\|X(s)-Y(s)\|^{2} \mathrm{~d} s \\
& \leq 2 M C^{2}(T+1) \int_{0}^{t} E\|X(s)-Y(s)\|^{2} \mathrm{~d} s .
\end{aligned}
$$

In addition, for any $a>0$ and $t \in[0, T]$, we have:

$$
\begin{aligned}
& \mathrm{e}^{-a t} E\|U(X)(t)-U(Y)(t)\|^{2} \\
& \leq 2 M C^{2}(T+1) \int_{0}^{t} \mathrm{e}^{-a(t-s)} \mathrm{e}^{-a s} E\|X(s)-Y(s)\|^{2} \mathrm{~d} s \\
& \leq 2 M C^{2}(T+1) \max _{0 \leq s \leq t} \mathrm{e}^{-a s} E\|X(s)-Y(s)\|^{2} \int_{0}^{t} \mathrm{e}^{-a(t-s)} \mathrm{d} s \\
& \leq 2 a^{-1} M C^{2}(T+1) \max _{0 \leq s \leq t} \mathrm{e}^{-a s} E\|X(s)-Y(s)\|^{2} .
\end{aligned}
$$

Therefore,

$$
\begin{aligned}
& \max _{0 \leq t \leq T}\left\{\mathrm{e}^{-a t} E\|U(X)(t)-U(Y)(t)\|^{2}\right\} \\
& \leq 2 a^{-1} M C^{2}(T+1) \max _{0 \leq s \leq T}\left\{\mathrm{e}^{-a s} E\|X(s)-Y(s)\|^{2}\right\} .
\end{aligned}
$$

Finally, if $a>2 M C^{2}(T+1)$, we have $U$ is contraction map in $Z$ respects to the norm

$$
\|X\|=\left\|X_{0}\right\|_{C_{r}}+\max _{0 \leq t \leq T}\left(\mathrm{e}^{-a t} E\|X(t)\|^{2}\right)^{1 / 2}, \quad X \in C_{a d} .
$$

Because this norm is equivalent to \|\|$_{a d}$, by applying fixed point principle we conclude that (1.1) has only mild solution on $[-r, T]$.

\section{REFERENCES}

[1] P. H. Bezandry and T. Diagana, "Almost Periodic Stochastic Processes,” Springer, Berlin, 2011. doi:10.1007/978-1-4419-9476-9

[2] N. M. Chuong and N. X. Thuan, "The Surjectivity of Semiregular Maximal Monotone Random Mappings," Random Operators and Stochastic Equations, Vol. 10, No. 1, 2002, pp. 13-24.

[3] N. M. Chuong and N. X. Thuan, "Random Equations for Weakly Semi-Monotone Operators of Type (S) and Semi J-Monotone Operators of Type (J-S)," Random Operators and Stochastic Equations, Vol. 10, No. 2, 2002, pp. 123132.

[4] N. M. Chuong and N. X. Thuan, "Random Equations for Semi H-Monotone Operators," Random Operators and Stochastic Equations, Vol. 10, No. 4, 2002, pp. 1-8.

[5] N. M. Chuong and N. X. Thuan, "Random Nonlinear Variational Inequalities for Mappings of Monotone Type in Banach Spaces," Stochastic Analysis and Applications, Vol. 24, No. 3, 2006, pp. 489-499. 
doi:10.1080/SAP-200064451

[6] N. M. Chuong and N. X. Thuan, "Random Fixed Point Theorems for Multivalued Nonlinear Mappings," Random Operators and Stochastic Equations, Vol. 9, No. 3, 2001, pp. 1-10.

[7] L. Gawarecki and V. Mandrekar, "Stochastic Differential
Equations in Infinite Dimensions,” Springer, Berlin, 2011. doi:10.1007/978-3-642-16194-0

[8] G. Da Prato and L. Tubaro, "Stochastic Partial Differential Equations and Applications, VII,” Chapman \& Hall/ CRC, New York, 2006. 